\title{
Attitude Expression of the Business Text and Its Translation Study Under the Appraisal Theory
}

\author{
Zhao Yushan*, Xu Yanan, Lv Liangqiu \\ School of Foreign Languages, North China Electric Power University, Beijing, China \\ Email address: \\ 15901356067@139.com (Zhao Yushan), Mier1452@163.com (Xu Yanan), 1lq2803@163.com (Lv Liangqiu) \\ *Corresponding author
}

\section{To cite this article:}

Zhao Yushan, Xu Yanan, Lv Liangqiu. Attitude Expression of the Business Text and Its Translation Study Under the Appraisal Theory. Science Innovation. Vol. 4, No. 1, 2016, pp. 11-15. doi: 10.11648/j.si.20160401.13

Received: February 24, 2016; Accepted: March 28, 2016; Published: April 8, 2016

\begin{abstract}
According to the Appraisal Theory put forward by Martin, this paper explores the attitude expression of the business text and its translation. There are several different kinds of business texts which have the same ways to express the textual attitude, including overt and covert presentation. Both overt and covert presentation can convey the positive and negative attitude of the text. While working out the translating work, translators need to know well the attitude of the source text first. And in the process of translation, it is necessary for translators to use corresponding words which can directly express overt attitude of the business text. As for covert attitude, translators need to figure out the implications from the ideational meaning of the words with the help of understanding the specific context. In addition, the translators also need to apply 'unfaithful' method to do trnslating work in order to meet the need of target-language readers.
\end{abstract}

Keywords: Appraisal Theory, Business Translation, Attitude Expression

\section{从评价理论看商务文本的态度表达及其翻译研究}

赵玉闪 ${ }^{*}$, 徐亚男, 吕亮球

外国语学院, 华北电力大学, 北京, 中国

邮箱

15901356067@139. com（赵玉闪），Mier1452@163.com（徐亚男），11q2803@163.com（吕亮球）

\begin{abstract}
摘要: 本文根据Matin提出的评价理论对商务文本的态度表达及其翻译进行探讨。商务文本有不同类型, 其态度表达的 方式都包括显性表达和隐性表达，不管哪种表达方式都能够传达积极或消极的态度意义。译者在翻译时，首先要把握 好源语文本的态度意义, 然后在翻译过程中使用相对应的表态词汇, 再现商务语篇显性态度。译者还需要挖掘词汇概 念意义隐含的态度意义, 结合具体语境理解并完成商务语篇的隐性态度的表达。另外, 译者还会采取 “不忠实性” 译 文来表达原文文本中的态度意义，满足目标语读者的需要。
\end{abstract}

关键词: 评价理论, 商务翻译, 态度表达

\section{1. 引言}

系统功能语言学的三大元功能包括概念功能、人际功 能和语篇功能, 其中语言学家们对于人际功能的研究越来
越深入。Matin的评价理论是就是在系统功能语法中人际 意义的研究中发展起来的, 该理论为语篇分析提供了很好 的分析框架。学者们将评价理论也逐渐运用于语篇分析与 翻译之中, 对语篇进行评价分析, 并且以此来指导翻译实 
践工作。张先刚利用评价理论对语篇翻译中的表态资源、 态度来源及语篇姿态进行了研究。另外, 很多学者将该理 论运用到具体语篇翻译的评价研究之中。陈明瑶将评价理 论运用于新闻语篇的分析与翻译之中, 新闻报道语篇渗透 了媒体的态度, 他认为要避免译者态度的介入。张美芳研 究了语言的评价意义及译者的价值取向, 认为由于价值取 向的不同, 会在一定程度上产生“不忠实”于原作的译文。 自中国改革开放以来, 商务活动不断增加。随着中国入世 以及全球化的发展, 中国与西方国家的商务交流及国际经 贸活动也越来越频繁, 商务翻译的重要性日益突出。因此, 商务翻译的研究受到了很多学者的关注。扶丽华利用评价 理论研究了商务语篇的翻译, 但主要是商务信函在语篇态 度上的显性和隐性表达。本文将从评价理论的角度对各种 商务文本的态度表达以及翻译效果进行探讨, 以期更好地 把握和传达语篇的态度意义, 指导商务翻译的实践工作, 尽可能地传递出商务文本的态度意义及信息。

\section{2. 评价理论及态度系统}

评价理论的发展基于韩礼德系统功能语法语言学, 是 人际功能理论的新发展及延伸。在系统功能语言学家门看 来, 说话人/作者的经验体会通过语言表达出来, 社会关 系以及社会身份的构建也会通过语言的表达实现, 在语言 的帮助下，语篇就会成为一个连贯的整体。语篇的产生过 程则反映了说话人/作者对这些经验的观点、态度和判断。 说话人通过人际功能闯入语境, 表达自己的态度和判断, 同时也寻求影响他人的态度和判断。人际功能表达了与情 景有关的角色关系 [1]。评价理论认为, 人们通过各种语 言表达, 展现出自己的主观态度、观点或判断, 进而建立 和维持双方的人际关系。汤普森 (Thompson) 认为, “评 价” 是 “所有语篇意义的核心问题，任何对语篇中的有关 人际功能的分析必然涉及到评价……评价是指说话者对 事物 (如人、事件、行为……观点等) 的看法, 通常有好 坏之分, 也有强弱之分” [2]。Matin对于评价理论的研究 较为深入而全面, 其建立的评价系统是由态度、介入和级 差三个系统组成的 [3]。这三个系统中又分别包含各自的 变量。其中, 态度由情感、判断和鉴赏三个变量组成, 它 揭示了文本语篇中语言使用者对描述对象的态度、观点和 立场 [4], 说话人/作者以各种价值作为判断手段, 使得情 感反应以及参与者和过程进行紧密的联系。情感是根据个 人的标准对其他事物进行的各种情绪的表达, 包括肯定和 否定的情感表达，具有很强的主观性。判断是根据一系列 社会制度规范对某个行为的肯定或否定的评价。我们会将 某件事物判断为合法/不合法、道德/不道德、社会可接受 /社会不可接受、正常/不正常。判断可分为两大类, 即社 会许可(social sanction)和社会尊严(social esteem), 这两大类别的判断同样具有肯定和否定两面的含义。鉴赏 是指根据美学标准对事物表达的看法, 包括反应 (reaction)、组成 (composition) 和评估 (valuation) 三个子范畴。评价系统详尽地将表态词汇进行了分类, 主 要是有关词汇项的。在态度子系统中, 主要是以各种文本、 行为以及现象来进行情感态度的表达, 并会运用词汇项来
进行行为的判断, 对事物依据审美标准以及社会价值来进 行鉴赏。随着学者们对于评价理论的研究, 评价理论也逐 渐从词汇层面上的研究转为语篇层面上的研究。

态度分析是语篇翻译中的一个重要环节。态度研究可 探讨语言使用者如何利用词汇资源对行为进行判断以及 对事物的价值进行鉴赏 [5]。做好语篇翻译的前提需要译 者能够揣摩并把握好源语文本的态度、立场与观点, 并能 将源语文本所要表达的情感意义尽可能地表达出来, 以避 免产生误解, 这样才能称之为一篇合格的翻译作品。评价 理论可以用于对源语文本的整个或部分语篇的态度表达 的分析, 还可以用于评估译出的目的语是否与源语文本的 态度表达相符合 [6]。商务文本中的态度意义不仅包括显 性态度的表达, 还包括隐性态度的表达。显性态度的表达 经由直译的方法, 借助表态词汇就可以直接将源语文本中 的态度表达出来。而由于语言文化上存在着, 因此译者在 做翻译时, 并不需要完全对原语文本进行词对词的翻译, 只要达到态度意义上的对等即可, 这就有利于在处理概念 意义时的隐性态度的表达。此外, 由于评价标度的存在以 及原作者与译者的价值观有所差别, 因此, 译者在理解、 翻译原文的过程中, 会渗透着自己的价值观, 从而引发“不 忠实” 译文的产生。本文针对商务文本的态度表达和翻译 进行探讨, 以态度方面的情感、裁决和鉴赏三个变量作为 分析的理论框架。

\section{3. 态度意义案例分析}

态度意义在表达方式上分为显性表达和隐性表达。显 性表达方式是通过明显的表态词汇来表达出明确的态度 和情感。翻译时, 译者可以借助带有感情色彩的形容词、 副词、动词、名词仔细揣摩源语文本的态度, 选择相应的 目的语, 来表达源语语篇的态度, 从而再现源语语篇的态 度意义。然而, 语篇中不含有表达态度的词汇并不代表无 法表达作者的态度, 概念意义的词汇仍可以将文本暗含的 态度表达出来, 只是没有表态词汇表达得那么明显。因此, 需要译者把握好词汇概念意义所蕴含的作者的态度, 将其 中的隐性态度展现出来 $[7]$ 。

\section{1. 使用相对应的表态词汇}

在商务翻译中, 译者首先要了解原语文本所表达的态 度, 再选择与之相对应的译入语, 使用相应的表态词汇进 行翻译, 这样使译语充分表达原语的态度, 再现商务语篇 显性态度。

(1) We will be obliged if you could quote US a favorable price。

贵公司如能报给我公司优惠价格, 我公司将十分感激。

(2) We are very much pleased/glad to receive your letter.

我公司非常高兴收到贵公司的信函。

(3) We regret to inform you that we are not in a position to supply you with such large demands. 我公司现在不能供应如此大量的需求, 甚兼。 
(4) We are sorry that we are not in a position to accept your offer.

我公司无法接受贵公司报盘, 请见谅。

(5) We have received your quotation for the commodity. We appreciate your prompt attention。

已收到你方关于商品的报价单, 非常感谢你方的快速 回复。

上面的举例是选取了商务信函中的五个例子，分别 含有各种词性的表态词汇。商务信函具有正确、简洁、 清楚、完整、具体、礼貌和体谅的特点 [8]。其中礼貌和 体谅的态度意义既可以通过表态词汇显性表达出来, 也 可以通过概念意义的词汇隐性表达出来。例 (1)，（2）

和 (5) 中通过appreciate, obliged, pleased, glad 等积极地表态词汇来表达愉悦的态度。而例（3），(4)

中, 则是通过regret, sorry等表态词汇来委婉地表达遗 憾的消极情感。对于令合作方不快的事项，应该使用委 婉语, 以获取对方的好感与肯定, 从而增进双方的合作 意愿, 避免交际过程中的冒昧, 也能体现商务信函礼貌 和体谅的特点。

(6) And the benefits arising from this work including water-proofness, precision and durability are the result of Rolex' s continuous pursuit of perfection. From the most elegant and prestigious models to the professional time pieces, all are exquisitely crafted.

而所有这些努力所带来的益处, 包括防水性、精确性 以及耐久性都是劳力士不断追求卓越的结果。劳力士优雅 尊贵, 专业精准, 每一块手表都做工精湛。

(7) Gold is cold. Diamonds are dead. A Limousine is a car. Don' t Pretend. Feel what's real. C' est Ca Que J'adore.

此时, 黄金显得冰冷, 钻石缺乏了生机, 豪华轿车也 不够吸引, 不要造作, 感受真实的奢华, 唯有, 迪奥真我 香水。

商业广告也是商务文本的一种形式。例（6）选取劳 力士手表的广告进行商务翻译的态度研究。源语文本中, 以名词water-proofness、precision、durability, 形容 词elegant and prestigious, professional、crafted, 副词exquisitely对劳力士手表的质量进行了鉴赏, 而译 文中使用了相应地鉴赏词汇对其进行评价。该广告为了更 有说服力, 基本没有使用情感词汇，而使用的鉴赏词汇较 多, 说明的其中的主观因素很少, 描述很客观。而译文很 好地选用了相应的表态词汇, 将广告中的显性态度表达出 来。例 (7) 是一则迪奥香水的广告, 该广告用形容词 cold, dead对黄金和钻石作了一定的鉴赏, 而对于Limousine (豪 华汽车) 的评价则是只是一辆普通的car。cold, dead和 car都充满了贬义, 从而与迪奥香水的 “真实的奢华” 形 成对比, 其目的在于强调该香水的品质和独特性。在这则 广告的翻译中, 译者同样使用了相应的表达态度的词汇, 将广告中的显性态度表达出来, 只是该译文还使用了增译 的方法进行编译, “奢华”一词的增译, 迎合了中国时尚 女性消费者的购物心理。

\section{2. 挖掘词汇概念意义隐含的态度意义}

商务文本的翻译中, 有些纯描写性质的词汇并没有以 一种显性的评价方式来表达原语文本的态度, 这些词汇并 不是很明晰的话语, 但却隐含着某种评价意义。译者在进 行翻译工作时, 需要挖掘出这些词汇概念意义中所要表达 的态度意义, 从而再现商务语篇隐性态度。

( 8 ) U.S. economist Nouriel Roubini, the Cassandra of the crisis, reckons that if all goes right and nothing terrible goes wrong, the global economy might grow nearly $4 \%$ this year.

美国经济学家Nouriel Roubini和希腊神话中能够预 言凶事的卡珊德拉一样, 能够预言危机的发生, 他认为, 如果一切顺利, 这些糟糕的事情就不会持续, 全球经济今 年可能就会上升将近 4 个百分点。

例（8）中的 “Cassandra” 原指希腊神话中能预卜凶 吉的却不被人相信的女预言师。将Nouriel Roubini比喻 成这个女预言师, 说明他的观点不被人相信, 也就是说其 他经济学家并没有看到全球经济复苏的迹象。原文中虽然 没有出现具体的表态词汇, 但作者通过词汇隐喻手段表达 了其中隐含的态度情感。而在翻译的时候, 为了将其中隐 含的态度意义表达出来, 译者可以采用转译、改译、释义 或增译等手段进行翻译, 这样才会有利于读者把握原文中 的态度意义, 最大程度地表达出原文的思想。

(9) Of course, that is precisely what the bailed out banks have done with their ostrich-sized nest eggs: tucked them away for a rainy day.

诚然, 那些得到救助的银行就是这样对待自己手中 “庞大的储备金” 的：留着防备更大的危机。

例（9）中, ostrich-sized nest eggs字面意思是 “沱 鸟大小的留窝蛋” , 引申义为 “庞大的储备金” 这里的意 思是表示银行并不发放用于借给需要贷款的人的资金, 而 是将这笔资金储存起来，以备未来更大的经济危机。这句 话中含有强烈的讽刺意味, 因为该资金就是用于发放到公 民手中以刺激经济, 解决经济危机的, 而银行却用以防备 未来的危机。虽然该语句中没有明显的表态词汇, 但是, 翻译时译者需要仔细环磨其中的隐喻义所具有的评价意 义, 以准确地再现其隐含的态度意义。“庞大的储备金” 一词加引号就很好地将其中的讽刺的隐含意义表达出来, 再现了作者的态度意义。

(10) Annual gross domestic product growth tumbled from a supercharged $8.3 \%$ to just $1.1 \%$-the most rapid decline since World War II. In fairness, given the pumped-up state of the stock market and the technology sector at the height of the boom, an ugly ending may well have been inevitable.

译文 1 : 年均国内生产总值从惊人的 $8.3 \%$ 狂跌至 1. $1 \%$ 一这是自二战以来最为神速的大滑坡。正所谓物极必 反, 股票市场和科技产业在经济鼎盛时期极度繁荣, 其可 悲可叹的结局也不可避免。

译文 $2:$ 年均国内生产总值从惊人的 $8.3 \%$ 狂跌至 1. $1 \%$ 一这是自二战以来最大的滑坡。股票市场和科技产业 
在经济鼎盛时期膨胀至极, 而其可悲可叹的结局也不可避 免, 正所谓物极必反。

例（10）中, rapid是一个中性词, 但是用来修饰 decline就有贬义的意味, 因此, 为了达到态度意义的对 等, 译者需要其中隐含的贬义表达出来, 因此, 使用 “神 速”一词实为不妥, 译为 “最大的滑坡” 则较为妥当。同 样, 在该语境中pumped-up一词也是具有贬义的意味, 译 为 “极度繁荣” 并不能体现出文中所说的in fairness (物 极必反）, 若译为 “膨胀至极” 则更为妥当。由此可以看 出, 译文 2 相对于译文 1 来说更为合适。两个译文的不同之 处主要在于个别词汇的 “襄贬” 含义的处理, 如果处理不 当, 则会造成意义的完全相反。朱永生 (2009) 认为, 在 评价研究中, 孤立的词汇所能起到的作用是有限的, 需要 将词汇和语境的研究密切结合起来, 这样才能对评价意义 的解读有一个较为全面的认识 [9]。针对这种词汇衰贬意 义的处理方法则是应该充分体会并挖掘原文文本所隐含 的意义, 在译文语篇中采用不同的语言资源灵活处理, 进 而将其隐含意义在译文中体现出来。

\section{3. 利用 “不忠实性” 译文表达态度意义}

态度意义的表达中, 不仅会有显性态度的表达和隐性 态度的表达, 译者还会采取 “不忠实性” 译文来表达原文 文本中的态度意义。

首先，由于正面与负面的评价之间存在着不同的评价 标度 (scale), 因此, 译者在翻译时会出现 “不忠实性” 译文的现象。例如, 同样是rich（富裕的）一词, 但在不 同的搭配关系、句型或上下文, 就会产生不同的译入语。 例如, rich achievement（卓越的成就）、rich clothes （华丽的衣服）、rich jewels（昂贵的珠宝）、rich furnitures（豪华的家具）中, 虽然都表达出了积极地态 度意义，但并没有使用忠实于原文而译为 “富裕的” , 这 就是由于对于不同的对象会有不同评价标度造成。

由于中西方文化的差异, 中西方对同一事物的思维方 式以及价值观都存在着巨大的差异, 这种文化差异仍然存 在于商务语篇翻译之中。评价是说话人/作者对事情进行 主观上的表达看法, 其中蕴含了说话人/作者的世界观、 人生观和价值观。译者在做翻译工作时, 不仅会考虑原文 中的态度意义, 还会将自己的价值观渗透于译文之中, 从 而会导致 “不忠实” 于原文的译文的产生。因此, 虽然翻 译讲究 “信达雅”, “忠实” 于原文是翻译的要求之一, 但在做商务文本的翻译分析的过程中, 为了符合目标语读 者的需求, 不仅要考虑原文文本所要表达的态度意义, 还 需要考虑针对于不同文本对象的评价标度以及译者的价 值观, 才能很好地评价译文的质量。

（11）酷暑难㥿, 人群熙攘之时, 您不妨合上双眼, 驰骋您的想像: 碧绿澄清 的泱決湖水, 清新凉爽的山间 气息, 还有山巅上的刀口幢避暑山庄……多么令人神往啊! 您可以去欧洲, 也可以去北美洲。您还可以去距热闹的新 加坡仅几小时 的印度尼西亚多巴湖, 享受那里 “水天一 色” 的风光。多巴湖可以避暑, 这是因为它在Sumatra山 巅, 海拔8000米。您可以滑水, 您可以冲浪, 您可以扬帆
远航; 您还可以轻荡双桨, 乘着一叶扁舟, 悠然自得地领 略沙莫尼亚岛上的巴塔克小村庄迷人的风情。

If the heat is unbearable, and the crowds are beginning to press in on you, just close your eyes. Now, imagine a vast blue lake. Cool, fresh mountain air, and alpine chalet. You could be in Europe or in North America. Or you could be just a few hours away from busy Singapore, relaxing on the shores of Indonesia' s beautiful lake. Toba escapes the heat because it' s high in the mountains of Sumatra, 8000 meters above the sea level. Try your hand at water skiing. Go windsurfing, or sailing. Take a leisurely cruise to the fascinating Batak villages on Samoris Island [10].

（12）饭店集中中国传统神韵与西方现代风格于一体, 庄重、富丽、典雅。大堂的八根镀金大柱交相辉映, 白色 大理石楼梯上方悬挂有巨幅中国古典绘画。

The hotel integrates the traditional Chinese spirit and the modern Western style, magnificent, and elegant. In the lobby stand 8 gilded columns brilliant and over the white marble staircases hang huge classical Chinese paintings[10].

例 11 和例 12 分别是一则旅游广告和企业广告, 在翻译 过程中, 除了要与原文对应之外, 还要根据目的语读者的 社会和文化等方面的差异进行一定调整, 从而满足目的语 读者的需求。中国的旅游宣传或公司宣传是通常会描述得 绘声绘色, 会使用大量的描绘性的词语将事物描写得很细 致。然而, 西方文化则是讲究简洁明了, 重点突出。因此, 需要译者去除繁文桭节, 选择较为简单的词, 保持英文译 文的简洁性, 将宣传的重点突出。例（11）中, “碧绿澄 清的泱泱湖水, 清新凉爽的山间气息”, “水天一色的风 光”, “轻荡双桨, 乘着一叶扁舟, 悠然自得地领略” 中, 译者在评价方面进行了一定程度的删减, 分别译为a vast blue lake、cool、fresh mountain air、beautiful lake、take a leisurely cruise to, 虽然没有忠实于原 文, 但这样变换表达方式, 既能将原文中的态度意义彰显 出来, 又能满足外国消费者的心理与价值观。同样, 在例 (12) 中, 存在着大量的描绘性的词汇, 译者在翻译时采 用了也是采用了 “不忠实性” 译文来体现态度意义的。其 中的 “中国传统神韵”，“庄重、富丽、典雅”，“交相 辉映” 分别译为 the traditional Chinese spirit, magnificent and elegant, brilliant。译者运用省略和 重组的方法将原作中的一些评价意义删除, 保留了主要信 息, 既能将主要的态度意义表达出来, 又能使得译文不那 么啰嗦繁琐, 符合英文描写的表达习惯。

\section{4. 结束语}

中西方商务交流的日益频繁使得商务文本翻译的角色 越来越重要。商务文本包括很多种类型, 比如商务信函、 商务广告、商务报刊等等。本文利用评价理论对这几种商 务文本的态度表达进行了分析研究, 以更好地把握源语文 
本的态度意义。在态度表达方式上, 既有显性表达, 也有 隐性表达。对于显性表达，译者可以通过明显的表态词汇 进行把握, 并将其中的态度意义以相应的词汇再现出来。 而隐性表达则是需要译者仔细揣摩语篇中的语境和表达概 念意义的隐喻词汇, 并用合适的表达方式将其中暗含的态 度意义再现出来。此外, 译者还需要依据标度以及不同的 价值观进行 “不忠实” 于原文的翻译, 从而满足目标语读 者的心理要求。利用评价理论进行商务翻译实践, 不仅能 够把握语篇中态度意义和传达语篇中的人际意义, 还能够 对比源语文本与译文的态度意义差异来评估译文的质量。

\section{致谢}

本文为中央高校基本科研业务费专项资金项目《西方 后现代主义及翻译研究》 (华北电力大学, 项目号: 2014 ZD25）的阶段性成果之一。

\section{参考文献}

［1］杨信彰. 人际视角下的评价理论和元话语理论: 张敬源, 彭 渏, 何伟. 系统功能语言学前沿动态一第八届中国系统功能
语言学学术活动周报告文集 [C]. 北京: 外语教学与研究出 版社, 2009: 145-150。

[2] Thompson, G. Introduction Functional Grammar [M]. London. (Edward) Amold, 1996.

[3] 朱永生, 严世清. 系统功能语言学再思考 $[\mathrm{M}]$. 上海: 复旦大 学出版社, 2011。

[4] 张敬源. 功能语言学与翻译研究 [M]. 北京: 外语教学与研究 出版社, 2010。

[5] 马伟林. 人际功能的拓展一评价系统述评 $[\mathrm{J}]$. 南京社会科 学, 2007, 6: 142-146。

[6] 张先刚. 评价理论对语篇翻译的启示 $[J]$. 外语教学, 2007, 6 : $33-36$ 。

[7] 扶丽华. 从评价理论看商务语篇态度的表达及翻译 $[\mathrm{J}]$. 中 国科技翻译，2010，1：28-31。

[8] 孟宏. 刍议商务英语函电的特点及其翻译 $[\mathrm{J}]$. 商业时代, 2009，17：128-129。

[9] 朱永生. 概念意义中的隐性评价 [J]. 外语教学, 2009, 4: $1-5$ 。

[10］董晓波. 商务英语翻译 [M]. 对外经贸大学出版社, 2011, 10。 